International Journal of Instrumentation and Control Systems (IJICS) Vol.2, No.2, April 2012

\title{
ACtive Controller Design for the ANTI-SYNCHRONIZATION OF HYPERCHAOTIC QI AND HYPERCHAOTIC JHA SYSTEMS
}

\author{
Sundarapandian Vaidyanathan ${ }^{1}$ \\ ${ }^{1}$ Research and Development Centre, Vel Tech Dr. RR \& Dr. SR Technical University \\ Avadi, Chennai-600 062, Tamil Nadu, INDIA \\ sundarvtu@gmail. com
}

\begin{abstract}
This paper derives new results for the anti-synchronization of identical hyperchaotic Qi systems (2008), identical hyperchaotic Jha systems (2007) and non-identical hyperchaotic Qi and hyperchaotic Jha systems. Active nonlinear control is the method adopted to achieve the anti-synchronization of the identical and different hyperchaotic Qi and Jha systems. Our stability results derived in this paper are established using Lyapunov stability theory. Since the Lyapunov exponents are not required for these calculations, the active nonlinear control method is effective and convenient to achieve anti-synchronization of the identical and different hyperchaotic Qi and hyperchaotic Jha systems. Numerical simulations are shown to validate and illustrate the effectiveness of the anti-synchronization results derived in this paper.
\end{abstract}

\section{KEYWORDS}

Chaos, Hyperchaos, Anti-Synchronization, Active Control, Hyperchaotic Qi System, Hyperchaotic Jha System.

\section{INTRODUCTION}

Chaotic systems are nonlinear dynamical systems that are highly sensitive to initial conditions. This sensitivity is popularly known as the butterfly effect [1]. Chaos is an interesting nonlinear phenomenon and has been extensively and intensively studied in the last two decades [1-23]. Chaos theory has been applied in many scientific disciplines such as Mathematics, Computer Science, Microbiology, Biology, Ecology, Economics, Population Dynamics and Robotics.

Hyperchaotic system is usually defined as a chaotic system with more than one positive Lyapunov exponent. The first hyperchaotic system was discovered by O.E. Rössler ([2], 1979). Since hyperchaotic system has the characteristics of high capacity, high security and high efficiency, it has the potential of broad applications in nonlinear circuits, secure communications, lasers, neural networks, biological systems and so on. Thus, the studies on hyperchaotic systems, viz. control, synchronization and circuit implementation are very challenging problems in the chaos literature [3].

In most of the chaos synchronization approaches, the master-slave or drive-response formalism has been used. If a particular chaotic system is called the master or drive system and another chaotic system is called the slave or response system, then the idea of anti-synchronization is to use the output of the master system to control the slave system so that the states of the slave DOI : $10.5121 /$ ijics.2012.2203 
system have the same amplitude but opposite signs as the states of the master system asymptotically.

In 1990, Pecora and Carroll [4] introduced a method to synchronize two identical chaotic systems and showed that it was possible for some chaotic systems to be completely synchronized. From then on, chaos synchronization has been widely explored in a variety of fields including physical

[5], chemical [6], ecological [7] systems, secure communications [8-10], etc.

Since the seminal work by Pecora and Carroll [4], a variety of impressive approaches have been proposed for the synchronization of chaotic systems such as OGY method [11], active control method [12-15], adaptive control method [16-20], backstepping method [21-22], sampled-data feedback synchronization method [23], time-delay feedback method [24], sliding mode control method [25-27], etc.

In this paper, we derive new results for the anti-synchronization for identical and different hyperchaotic Qi and Jha systems using active nonlinear control. Explicitly, using active nonlinear control and Lyapunov stability theory, we achieve anti-synchronization for identical hyperchaotic Qi systems ([28], 2008), identical hyperchaotic Jha systems ([29], 2007) and non-identical hyperchaotic Qi and hyperchaotic Jha systems.

This paper has been organized as follows. In Section 2, we give the problem statement and our methodology. In Section 3, we give a description of the hyperchaotic Qi and Jha systems. In Section 4, we discuss the anti-synchronization of two identical hyperchaotic Qi systems. In Section 5, we discuss the anti-synchronization of two identical hyperchaotic Jha systems ([29], 2007). In Section 6, we discuss the anti-synchronization of non-identical hyperchaotic Qi and Jha systems. In Section 7, we summarize the main results of this paper.

\section{Problem Statement AND OUR Methodology}

Consider the chaotic system described by the dynamics

$$
\dot{x}=A x+f(x)
$$

where $x \in R^{n}$ is the state of the system, $A$ is the $n \times n$ matrix of the system parameters and $f: R^{n} \rightarrow R^{n}$ is the nonlinear part of the system. We consider the system (1) as the master or drive system.

As the slave or response system, we consider the following chaotic system described by the dynamics

$$
\dot{y}=B y+g(y)+u
$$

where $y \in R^{n}$ is the state of the system, $B$ is the $n \times n$ matrix of the system parameters, $g: R^{n} \rightarrow R^{n}$ is the nonlinear part of the system and $u \in R^{n}$ is the active controller of the slave system.

If $A=B$ and $f=g$, then $x$ and $y$ are the states of two identical chaotic systems. If $A \neq B$ or $f \neq g$, then $x$ and $y$ are the states of two different chaotic systems. 
In the active control method, we design a feedback controller $u$, which anti-synchronizes the states of the master system (1) and the slave system (2) for all initial conditions $x(0), y(0) \in R^{n}$.

If we define the anti-synchronization error as

$$
e=y+x
$$

then the error dynamics is obtained as

$$
\dot{e}=B y+A x+g(y)+f(x)+u
$$

Thus, the anti-synchronization problem is essentially to find a feedback controller $u$ so as to stabilize the error dynamics (4) for all initial conditions $e(0) \in R^{n}$.

Hence, we find a feedback controller $u$ so that

$$
\lim _{t \rightarrow \infty}\|e(t)\|=0 \text { for all } e(0) \in R^{n}
$$

We take as a candidate Lyapunov function

$$
V(e)=e^{T} P e,
$$

where $P$ is a positive definite matrix.

Note that $V: R^{n} \rightarrow R$ is a positive definite function by construction.

We assume that the parameters of the master and slave system are known and that the states of both systems (1) and (2) are measurable.

If we find a feedback controller $u$ so that

$$
\dot{V}(e)=-e^{T} Q e,
$$

where $Q$ is a positive definite matrix, then $\dot{V}: R^{n} \rightarrow R$ is a negative definite function.

Thus, by Lyapunov stability theory [30], the error dynamics (4) is globally exponentially stable and hence the condition (5) will be satisfied. Hence, the states of the master system (1) and the slave system (2) will be globally and exponentially anti-synchronized.

\section{SYSTEMS DESCRIPTION}

In this section, we describe the hyperchaotic systems studied in this paper, viz. hyperchaotic Qi system ([28], 2008) and hyperchaotic Jha system ([29], 2007).

The hyperchaotic Qi system ([28], 2008) is described by the dynamics 


$$
\begin{aligned}
& \dot{x}_{1}=a\left(x_{2}-x_{1}\right)+x_{2} x_{3} \\
& \dot{x}_{2}=b\left(x_{1}+x_{2}\right)-x_{1} x_{3} \\
& \dot{x}_{3}=-c x_{3}-\varepsilon x_{4}+x_{1} x_{2} \\
& \dot{x}_{4}=-d x_{4}+f x_{3}+x_{1} x_{2}
\end{aligned}
$$

where $x_{1}, x_{2}, x_{3}, x_{4}$ are the states and $a, b, c, d, \varepsilon, f$ are constant, positive parameters of the system.

The Qi system (8) exhibits a hyperchaotic attractor (see Figure 1), when the parameter values are taken as

$$
a=50, \quad b=24, \quad c=13, \quad d=8, \quad \varepsilon=33, f=30
$$
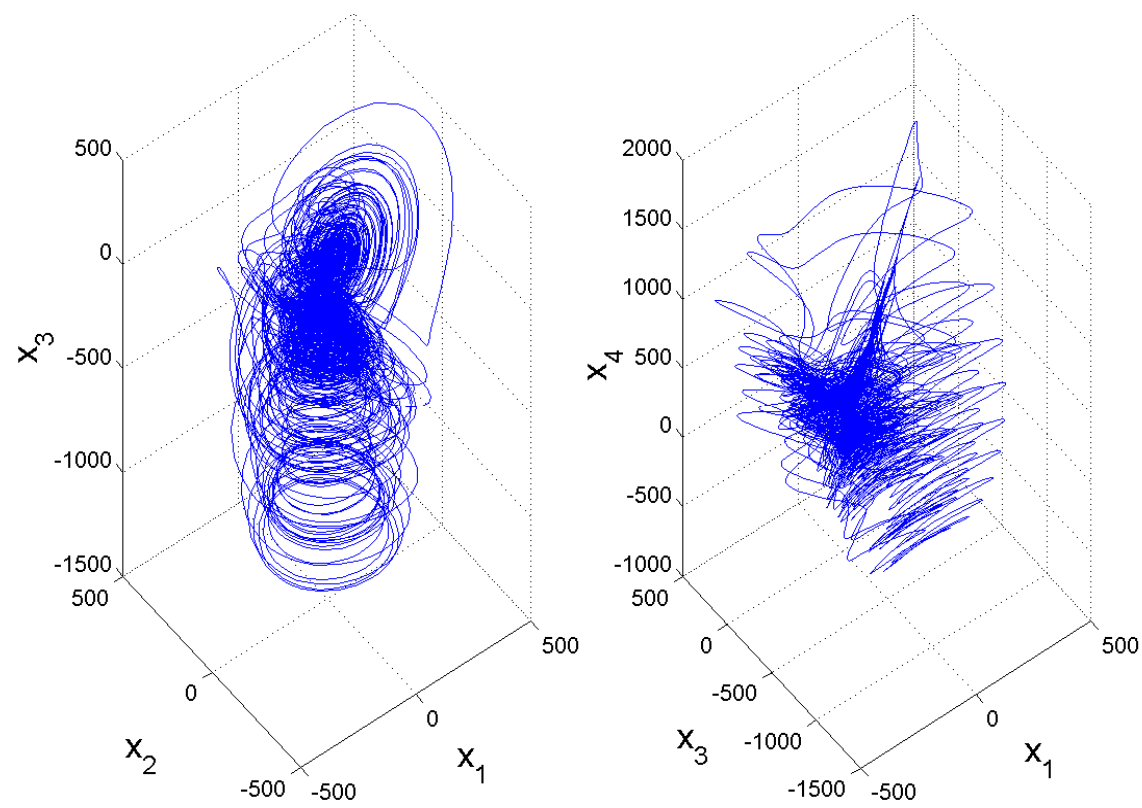

Figure 1. The Phase Portrait of the Hyperchaotic Qi System

The hyperchaotic Jha system ([29], 2007) is described by the dynamics

$$
\begin{aligned}
& \dot{x}_{1}=\alpha\left(x_{2}-x_{1}\right)+x_{4} \\
& \dot{x}_{2}=-x_{1} x_{3}+\beta x_{1}-x_{2} \\
& \dot{x}_{3}=x_{1} x_{2}-\gamma x_{3} \\
& \dot{x}_{4}=-x_{1} x_{3}+\delta x_{4}
\end{aligned}
$$

where $x_{1}, x_{2}, x_{3}, x_{4}$ are the states and $\alpha, \beta, \gamma, \delta$ are constant, positive parameters of the system. 
International Journal of Instrumentation and Control Systems (IJICS) Vol.2, No.2, April 2012

The Jha dynamics (10) exhibits a hyperchaotic attractor (see Figure 2), when the parameter values are taken as

$$
\alpha=10, \quad \beta=28, \quad \gamma=8 / 3, \quad \delta=1.3
$$
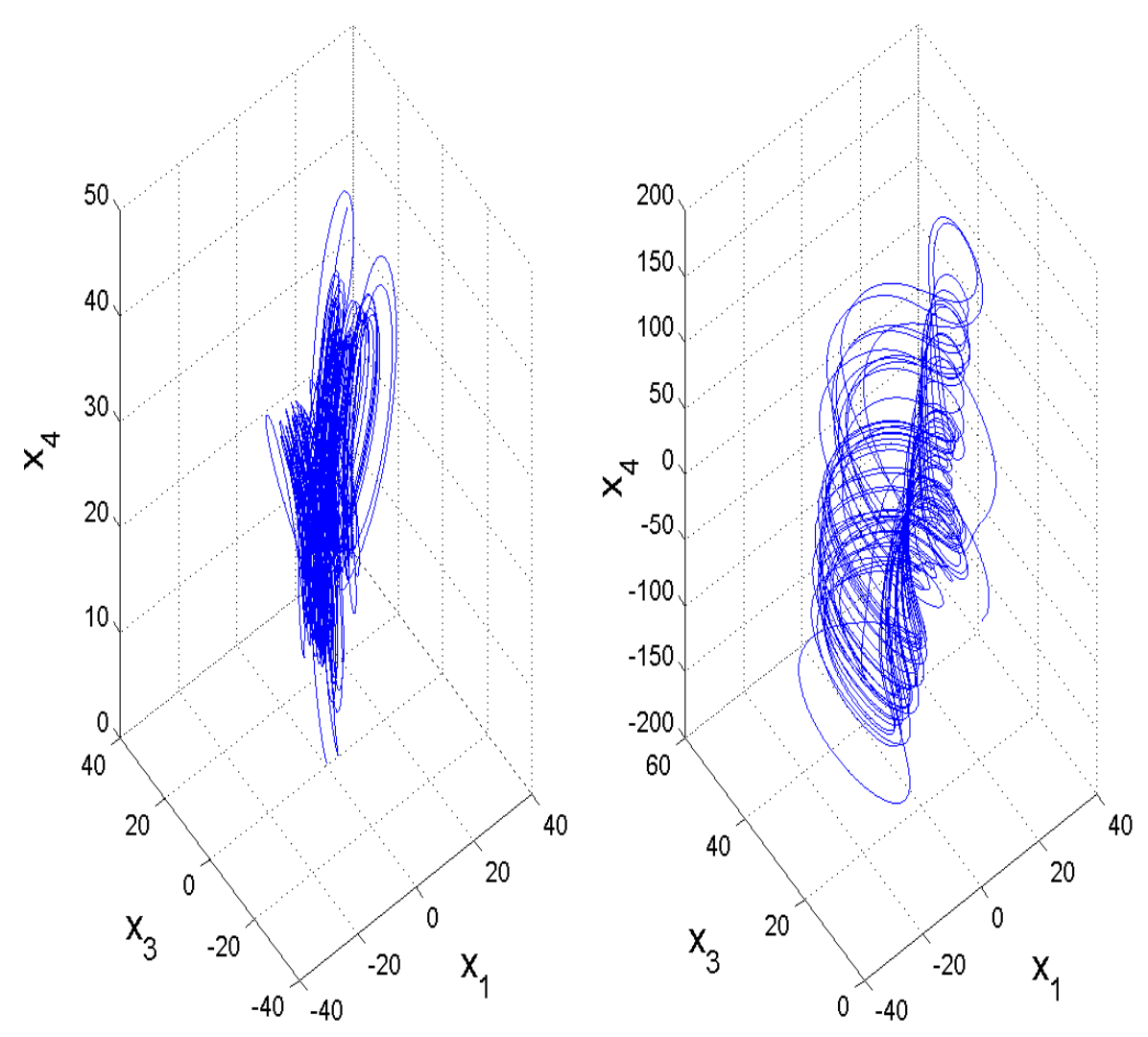

Figure 2. The Phase Portrait of the Hyperchaotic Jha System

\section{ANTI-SyNChronizATION OF IDENTICAL HYPERCHAOTIC QI SYSTEMS BY ACTIVE CONTROL}

\subsection{Theoretical Results}

In this section, we apply the active nonlinear control method for the anti-synchronization of two identical hyperchaotic Qi systems (2008).

Thus, the master system is described by the hyperchaotic Qi dynamics 


$$
\begin{aligned}
& \dot{x}_{1}=a\left(x_{2}-x_{1}\right)+x_{2} x_{3} \\
& \dot{x}_{2}=b\left(x_{1}+x_{2}\right)-x_{1} x_{3} \\
& \dot{x}_{3}=-c x_{3}-\varepsilon x_{4}+x_{1} x_{2} \\
& \dot{x}_{4}=-d x_{4}+f x_{3}+x_{1} x_{2}
\end{aligned}
$$

where $x_{1}, x_{2}, x_{3}, x_{4}$ are the state variables and $a, b, c, d, \varepsilon, f$ are positive parameters of the system. The slave system is described by the controlled hyperchaotic Qi dynamics

$$
\begin{aligned}
& \dot{y}_{1}=a\left(y_{2}-y_{1}\right)+y_{2} y_{3}+u_{1} \\
& \dot{y}_{2}=b\left(y_{1}+y_{2}\right)-y_{1} y_{3}+u_{2} \\
& \dot{y}_{3}=-c y_{3}-\varepsilon y_{4}+y_{1} y_{2}+u_{3} \\
& \dot{y}_{4}=-d y_{4}+f y_{3}+y_{1} y_{2}+u_{4}
\end{aligned}
$$

where $y_{1}, y_{2}, y_{3}, y_{4}$ are the state variables and $u_{1}, u_{2}, u_{3}, u_{4}$ are the active nonlinear controls to be designed.

The anti-synchronization error $e$ is defined by

$$
e_{i}=y_{i}+x_{i}, \quad(i=1,2,3,4)
$$

The error dynamics is obtained as

$$
\begin{aligned}
& \dot{e}_{1}=a\left(e_{2}-e_{1}\right)+y_{2} y_{3}+x_{2} x_{3}+u_{1} \\
& \dot{e}_{2}=b\left(e_{1}+e_{2}\right)-y_{1} y_{3}-x_{1} x_{3}+u_{2} \\
& \dot{e}_{3}=-c e_{3}-\varepsilon e_{4}+y_{1} y_{2}+x_{1} x_{2}+u_{3} \\
& \dot{e}_{4}=-d e_{4}+f e_{3}+y_{1} y_{2}+x_{1} x_{2}+u_{4}
\end{aligned}
$$

We choose the active nonlinear controller as

$$
\begin{aligned}
& u_{1}=-a\left(e_{2}-e_{1}\right)-y_{2} y_{3}-x_{2} x_{3}-k_{1} e_{1} \\
& u_{2}=-b\left(e_{1}+e_{2}\right)+y_{1} y_{3}+x_{1} x_{3}-k_{2} e_{2} \\
& u_{3}=c e_{3}+\varepsilon e_{4}-y_{1} y_{2}-x_{1} x_{2}-k_{3} e_{3} \\
& u_{4}=d e_{4}-f e_{3}-y_{1} y_{2}-x_{1} x_{2}-k_{4} e_{4}
\end{aligned}
$$

where the gains $k_{i},(i=1,2,3,4)$ are positive constants.

Substituting (16) into (15), the error dynamics simplifies to 


$$
\begin{aligned}
& \dot{e}_{1}=-k_{1} e_{1} \\
& \dot{e}_{2}=-k_{2} e_{2} \\
& \dot{e}_{3}=-k_{3} e_{3} \\
& \dot{e}_{4}=-k_{4} e_{4}
\end{aligned}
$$

Next, we prove the following result.

Theorem 4.1. The identical hyperchaotic Qi systems (12) and (13) are globally and exponentially anti-synchronized for all initial conditions with the active nonlinear controller defined by (16).

Proof. We consider the quadratic Lyapunov function defined by

$$
V(e)=\frac{1}{2} e^{T} e=\frac{1}{2}\left(e_{1}^{2}+e_{2}^{2}+e_{3}^{2}+e_{4}^{2}\right)
$$

which is a positive definite function on $R^{4}$.

Differentiating (18) along the trajectories of (17), we get

$$
\dot{V}(e)=-k_{1} e_{1}^{2}-k_{2} e_{2}^{2}-k_{3} e_{3}^{2}-k_{4} e_{4}^{2}
$$

which is a negative definite function on $R^{4}$.

Thus, by Lyapunov stability theory [30], the error dynamics (17) is globally exponentially stable.

Hence, the identical hyperchaotic Qi systems (12) and (13) are globally and exponentially anti-synchronized for all initial conditions with the active controller defined by (16).

This completes the proof.

\subsection{Numerical Results}

For simulations, the fourth-order Runge-Kutta method with time-step $h=10^{-8}$ is used to solve the differential equations (12) and (13) with the active nonlinear controller (16).

The feedback gains used in the equation (16) are chosen as

$$
k_{1}=5, k_{2}=5, k_{3}=5, k_{4}=5
$$

The parameters of the hyperchaotic Qi systems are chosen as

$$
a=50, \quad b=24, \quad c=13, \quad d=8, \quad \varepsilon=33, \quad f=30
$$

The initial conditions of the master system (12) are chosen as

$$
x_{1}(0)=8, \quad x_{2}(0)=26, \quad x_{3}(0)=-20, \quad x_{4}(0)=-15
$$


International Journal of Instrumentation and Control Systems (IJICS) Vol.2, No.2, April 2012

The initial conditions of the slave system (13) are chosen as

$$
y_{1}(0)=30, \quad y_{2}(0)=-5, \quad y_{3}(0)=15, \quad y_{4}(0)=-22
$$

Figure 3 shows the anti-synchronization of the identical hyperchaotic Qi systems.

Figure 4 shows the time-history of the anti-synchronization errors $e_{1}, e_{2}, e_{3}, e_{4}$.
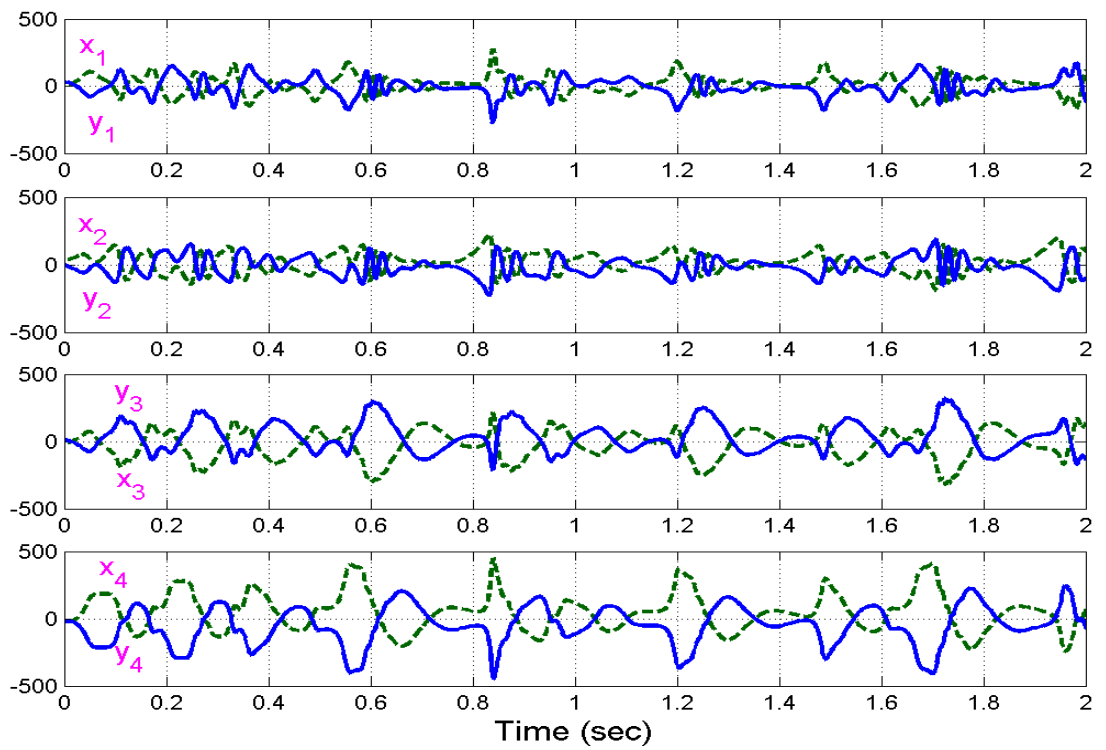

Figure 3. Anti-Synchronization of the Identical Hyperchaotic Qi Systems 
International Journal of Instrumentation and Control Systems (IJICS) Vol.2, No.2, April 2012

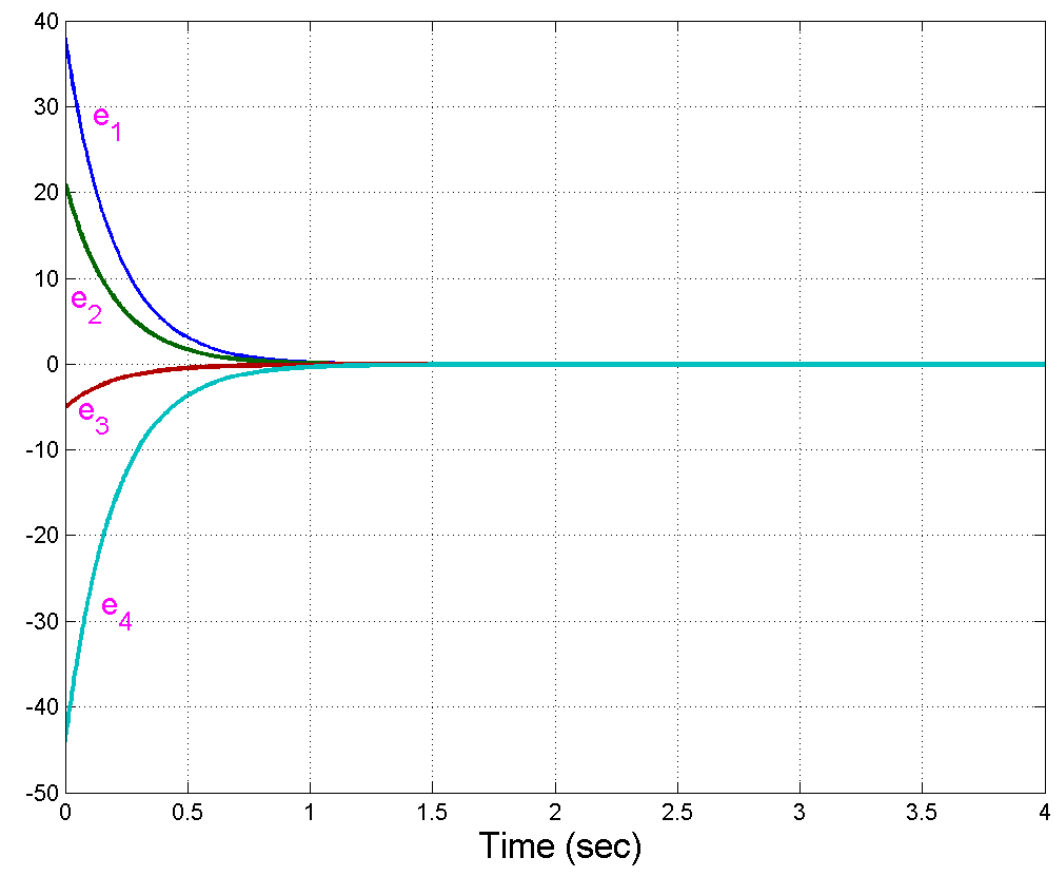

Figure 4. Time-History of the Anti-Synchronization Error

\section{ANTi- SynChronization of Identical Hyperchaotic Jha Systems BY ACTIVE CONTROL}

\subsection{Theoretical Results}

In this section, we apply the active nonlinear control method for the anti-synchronization of two identical hyperchaotic Jha systems (2007). Thus, the master system is described by the hyperchaotic Jha dynamics

$$
\begin{aligned}
& \dot{x}_{1}=\alpha\left(x_{2}-x_{1}\right)+x_{4} \\
& \dot{x}_{2}=-x_{1} x_{3}+\beta x_{1}-x_{2} \\
& \dot{x}_{3}=x_{1} x_{2}-\gamma x_{3} \\
& \dot{x}_{4}=-x_{1} x_{3}+\delta x_{4}
\end{aligned}
$$

where $x_{1}, x_{2}, x_{3}, x_{4}$ are the state variables and $\alpha, \beta, \gamma, \delta$ are positive parameters of the system.

The slave system is described by the controlled hyperchaotic Jha dynamics 


$$
\begin{aligned}
& \dot{y}_{1}=\alpha\left(y_{2}-y_{1}\right)+y_{4}+u_{1} \\
& \dot{y}_{2}=-y_{1} y_{3}+\beta y_{1}-y_{2}+u_{2} \\
& \dot{y}_{3}=y_{1} y_{2}-\gamma y_{3}+u_{3} \\
& \dot{y}_{4}=-y_{1} y_{3}+\delta y_{4}+u_{4}
\end{aligned}
$$

where $y_{1}, y_{2}, y_{3}, y_{4}$ are the state variables and $u_{1}, u_{2}, u_{3}, u_{4}$ are the active nonlinear controls to be designed.

The anti-synchronization error $e$ is defined by

$$
e_{i}=y_{i}+x_{i}, \quad(i=1,2,3,4)
$$

The error dynamics is obtained as

$$
\begin{aligned}
& \dot{e}_{1}=\alpha\left(e_{2}-e_{1}\right)+e_{4}+u_{1} \\
& \dot{e}_{2}=\beta e_{1}-e_{2}-y_{1} y_{3}-x_{1} x_{3}+u_{2} \\
& \dot{e}_{3}=-\gamma e_{3}+y_{1} y_{2}+x_{1} x_{2}+u_{3} \\
& \dot{e}_{4}=\delta e_{4}-y_{1} y_{3}-x_{1} x_{3}+u_{4}
\end{aligned}
$$

We choose the active nonlinear controller as

$$
\begin{aligned}
& u_{1}=-\alpha\left(e_{2}-e_{1}\right)-e_{4}-k_{1} e_{1} \\
& u_{2}=-\beta e_{1}+e_{2}+y_{1} y_{3}+x_{1} x_{3}-k_{2} e_{2} \\
& u_{3}=\gamma e_{3}-y_{1} y_{2}-x_{1} x_{2}-k_{3} e_{3} \\
& u_{4}=-\delta e_{4}+y_{1} y_{3}+x_{1} x_{3}-k_{4} e_{4}
\end{aligned}
$$

where the gains $k_{i},(i=1,2,3,4)$ are positive constants.

Substituting (24) into (23), the error dynamics simplifies to

$$
\begin{aligned}
& \dot{e}_{1}=-k_{1} e_{1} \\
& \dot{e}_{2}=-k_{2} e_{2} \\
& \dot{e}_{3}=-k_{3} e_{3} \\
& \dot{e}_{4}=-k_{4} e_{4}
\end{aligned}
$$

Next, we prove the following result.

Theorem 5.1. The identical hyperchaotic Jha systems (20) and (21) are globally and exponentially anti-synchronized for all initial conditions with the active nonlinear controller defined by (24).

Proof. We consider the quadratic Lyapunov function defined by 


$$
V(e)=\frac{1}{2} e^{T} e=\frac{1}{2}\left(e_{1}^{2}+e_{2}^{2}+e_{3}^{2}+e_{4}^{2}\right)
$$

which is a positive definite function on $R^{4}$.

Differentiating (26) along the trajectories of (25), we get

$$
\dot{V}(e)=-k_{1} e_{1}^{2}-k_{2} e_{2}^{2}-k_{3} e_{3}^{2}-k_{4} e_{4}^{2}
$$

which is a negative definite function on $R^{4}$.

Thus, by Lyapunov stability theory [30], the error dynamics (25) is globally exponentially stable.

Hence, the identical hyperchaotic Jha systems (20) and (21) are globally and exponentially antisynchronized for all initial conditions with the nonlinear controller defined by (24).

This completes the proof.

\subsection{Numerical Results}

For simulations, the fourth-order Runge-Kutta method with time-step $h=10^{-8}$ is used to solve the differential equations (20) and (21) with the active nonlinear controller (24).

The feedback gains used in the equation (24) are chosen as

$$
k_{1}=5, k_{2}=5, \quad k_{3}=5, \quad k_{4}=5
$$

The parameters of the hyperchaotic Jha systems are chosen as

$$
\alpha=10, \quad \beta=28, \quad \gamma=8 / 3, \quad \delta=1.3
$$

The initial conditions of the master system (20) are chosen as

$$
x_{1}(0)=8, \quad x_{2}(0)=20, \quad x_{3}(0)=18, \quad x_{4}(0)=4
$$

The initial conditions of the slave system (21) are chosen as

$$
y_{1}(0)=-18, \quad y_{2}(0)=24, y_{3}(0)=-11, \quad y_{4}(0)=22
$$

Figure 5 shows the anti-synchronization of the identical hyperchaotic Jha systems.

Figure 6 shows the time-history of the anti-synchronization errors $e_{1}, e_{2}, e_{3}, e_{4}$. 

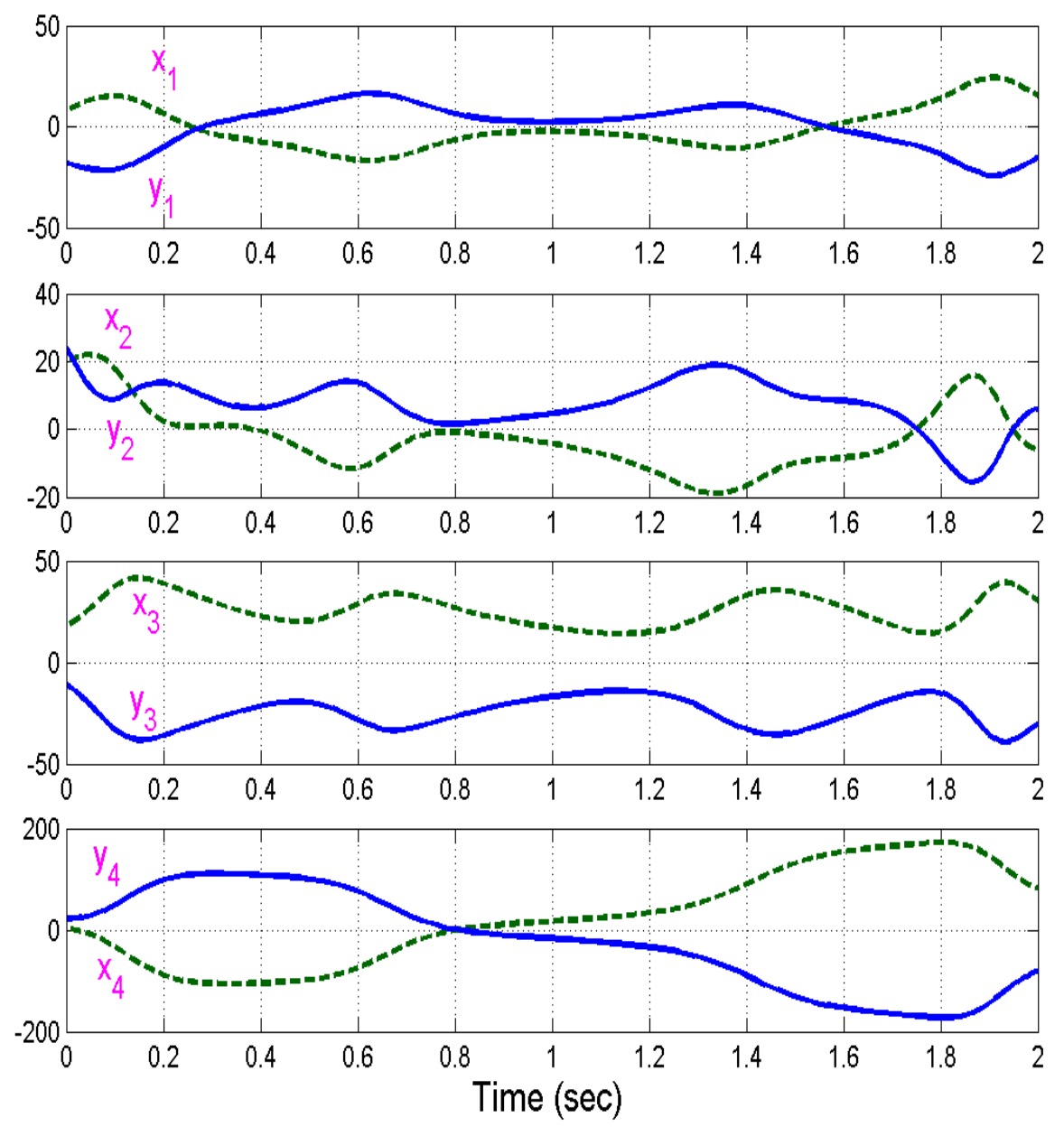

Figure 5. Anti-Synchronization of the Identical Hyperchaotic Jha Systems 
International Journal of Instrumentation and Control Systems (IJICS) Vol.2, No.2, April 2012

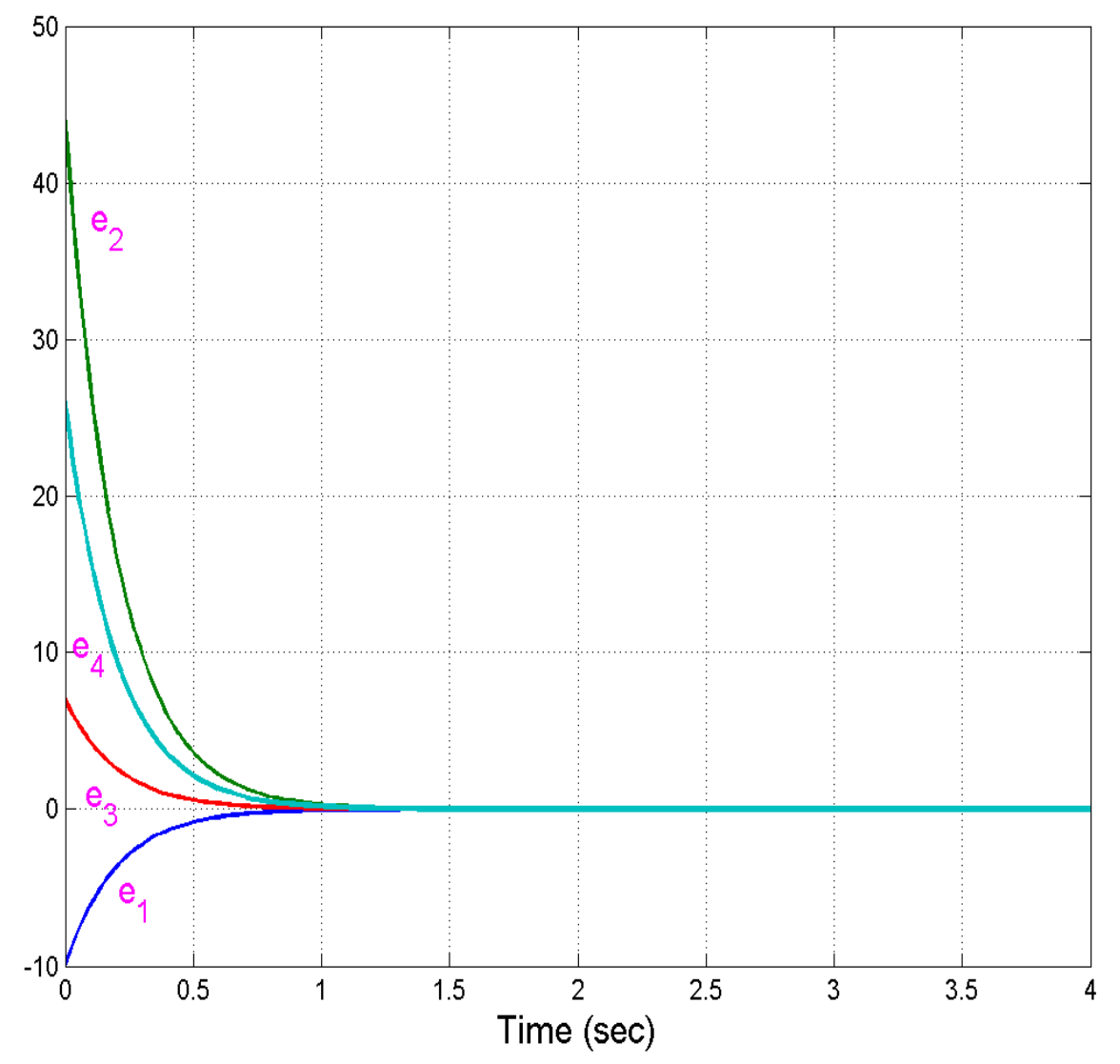

Figure 6. Time-History of the Anti-Synchronization Error

\section{ANTI- Synchronization of Non-IDENTICAL HyPERCHAOTIC QI AND HyPERCHAOTIC JHA SySTEMS BY ACTIVE CONTROL}

\subsection{Theoretical Results}

In this section, we apply the active nonlinear control method for the anti-synchronization of the non-identical hyperchaotic Qi system (2008) and hyperchaotic Jha system (2007).

Thus, the master system is described by the hyperchaotic Qi dynamics

$$
\begin{aligned}
& \dot{x}_{1}=a\left(x_{2}-x_{1}\right)+x_{2} x_{3} \\
& \dot{x}_{2}=b\left(x_{1}+x_{2}\right)-x_{1} x_{3} \\
& \dot{x}_{3}=-c x_{3}-\varepsilon x_{4}+x_{1} x_{2} \\
& \dot{x}_{4}=-d x_{4}+f x_{3}+x_{1} x_{2}
\end{aligned}
$$

where $x_{1}, x_{2}, x_{3}, x_{4}$ are the state variables and $a, b, c, d, \varepsilon, f$ are positive parameters of the system. 
The slave system is described by the controlled hyperchaotic Jha dynamics

$$
\begin{aligned}
& \dot{y}_{1}=\alpha\left(y_{2}-y_{1}\right)+y_{4}+u_{1} \\
& \dot{y}_{2}=-y_{1} y_{3}+\beta y_{1}-y_{2}+u_{2} \\
& \dot{y}_{3}=y_{1} y_{2}-\gamma y_{3}+u_{3} \\
& \dot{y}_{4}=-y_{1} y_{3}+\delta y_{4}+u_{4}
\end{aligned}
$$

where $y_{1}, y_{2}, y_{3}, y_{4}$ are the state variables, $\alpha, \beta, \gamma, \delta$ are positive parameters and $u_{1}, u_{2}, u_{3}, u_{4}$ are the active nonlinear controls to be designed.

The anti-synchronization error $e$ is defined by

$$
e_{i}=y_{i}+x_{i}, \quad(i=1,2,3,4)
$$

The error dynamics is obtained as

$$
\begin{aligned}
& \dot{e}_{1}=\alpha\left(y_{2}-y_{1}\right)+y_{4}+a\left(x_{2}-x_{1}\right)+x_{2} x_{3}+u_{1} \\
& \dot{e}_{2}=\beta y_{1}-y_{2}+b\left(x_{1}+x_{2}\right)-y_{1} y_{3}-x_{1} x_{3}+u_{2} \\
& \dot{e}_{3}=-\gamma y_{3}-c x_{3}-\varepsilon x_{4}+y_{1} y_{2}+x_{1} x_{2}+u_{3} \\
& \dot{e}_{4}=\delta y_{4}-d x_{4}+f x_{3}-y_{1} y_{3}+x_{1} x_{2}+u_{4}
\end{aligned}
$$

We choose the active nonlinear controller as

$$
\begin{aligned}
& u_{1}=-\alpha\left(y_{2}-y_{1}\right)-y_{4}-a\left(x_{2}-x_{1}\right)-x_{2} x_{3}-k_{1} e_{1} \\
& u_{2}=-\beta y_{1}+y_{2}-b\left(x_{1}+x_{2}\right)+y_{1} y_{3}+x_{1} x_{3}-k_{2} e_{2} \\
& u_{3}=\gamma y_{3}+c x_{3}+\varepsilon x_{4}-y_{1} y_{2}-x_{1} x_{2}-k_{3} e_{3} \\
& u_{4}=-\delta y_{4}+d x_{4}-f x_{3}+y_{1} y_{3}-x_{1} x_{2}-k_{4} e_{4}
\end{aligned}
$$

where the gains $k_{i},(i=1,2,3,4)$ are positive constants.

Substituting (32) into (31), the error dynamics simplifies to

$$
\begin{aligned}
& \dot{e}_{1}=-k_{1} e_{1} \\
& \dot{e}_{2}=-k_{2} e_{2} \\
& \dot{e}_{3}=-k_{3} e_{3} \\
& \dot{e}_{4}=-k_{4} e_{4}
\end{aligned}
$$

Next, we prove the following result.

Theorem 6.1. The non-identical hyperchaotic Qi system (28) and hyperchaotic Jha system (29) are globally and exponentially anti-synchronized for all initial conditions with the active nonlinear controller defined by (32). 
Proof. We consider the quadratic Lyapunov function defined by

$$
V(e)=\frac{1}{2} e^{T} e=\frac{1}{2}\left(e_{1}^{2}+e_{2}^{2}+e_{3}^{2}+e_{4}^{2}\right)
$$

which is a positive definite function on $R^{4}$.

Differentiating (34) along the trajectories of (33), we get

$$
\dot{V}(e)=-k_{1} e_{1}^{2}-k_{2} e_{2}^{2}-k_{3} e_{3}^{2}-k_{4} e_{4}^{2}
$$

which is a negative definite function on $R^{4}$.

Thus, by Lyapunov stability theory [30], the error dynamics (33) is globally exponentially stable.

Hence, the non- identical hyperchaotic Qi system (28) and hyperchaotic Jha system (29) are globally and exponentially anti-synchronized for all initial conditions with the active nonlinear controller defined by (32). This completes the proof.

\subsection{Numerical Results}

For simulations, the fourth-order Runge-Kutta method with time-step $h=10^{-8}$ is used to solve the differential equations (28) and (29) with the active nonlinear controller (32).

The feedback gains used in the equation (32) are chosen as

$$
k_{1}=5, k_{2}=5, k_{3}=5, k_{4}=5
$$

The parameters of the hyperchaotic Qi systems are chosen as

$$
a=50, \quad b=24, \quad c=13, \quad d=8, \quad \varepsilon=33, \quad f=30
$$

The parameters of the hyperchaotic Jha systems are chosen as

$$
\alpha=10, \quad \beta=28, \quad \gamma=8 / 3, \quad \delta=1.3
$$

The initial conditions of the master system (28) are chosen as

$$
x_{1}(0)=-25, \quad x_{2}(0)=15, \quad x_{3}(0)=18, \quad x_{4}(0)=-6
$$

The initial conditions of the slave system (29) are chosen as

$$
y_{1}(0)=8, \quad y_{2}(0)=24, \quad y_{3}(0)=-7, \quad y_{4}(0)=33
$$

Figure 7 shows the anti-synchronization of the hyperchaotic Qi and hyperchaotic Jha systems.

Figure 8 shows the time-history of the synchronization errors $e_{1}, e_{2}, e_{3}, e_{4}$. 

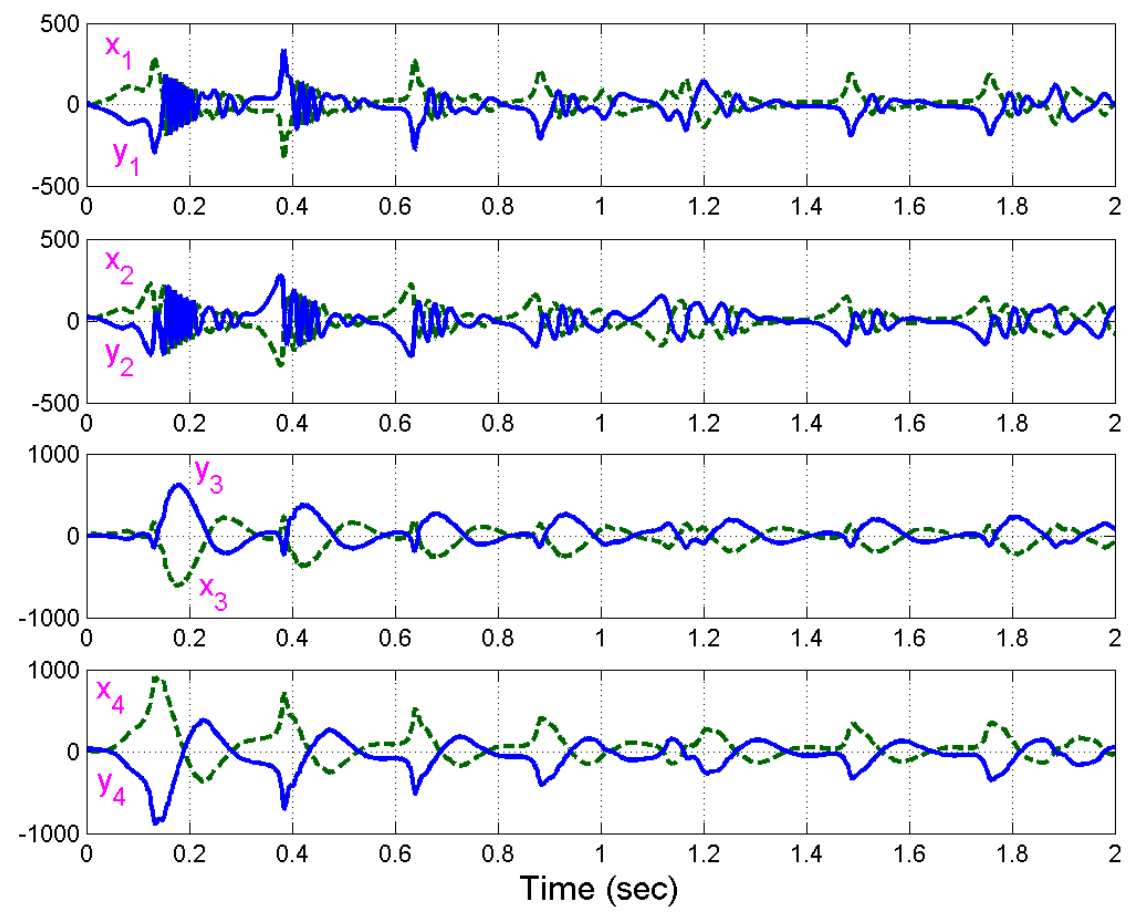

Figure 7. Anti-Synchronization of Hyperchaotic Qi and Hyperchaotic Jha Systems

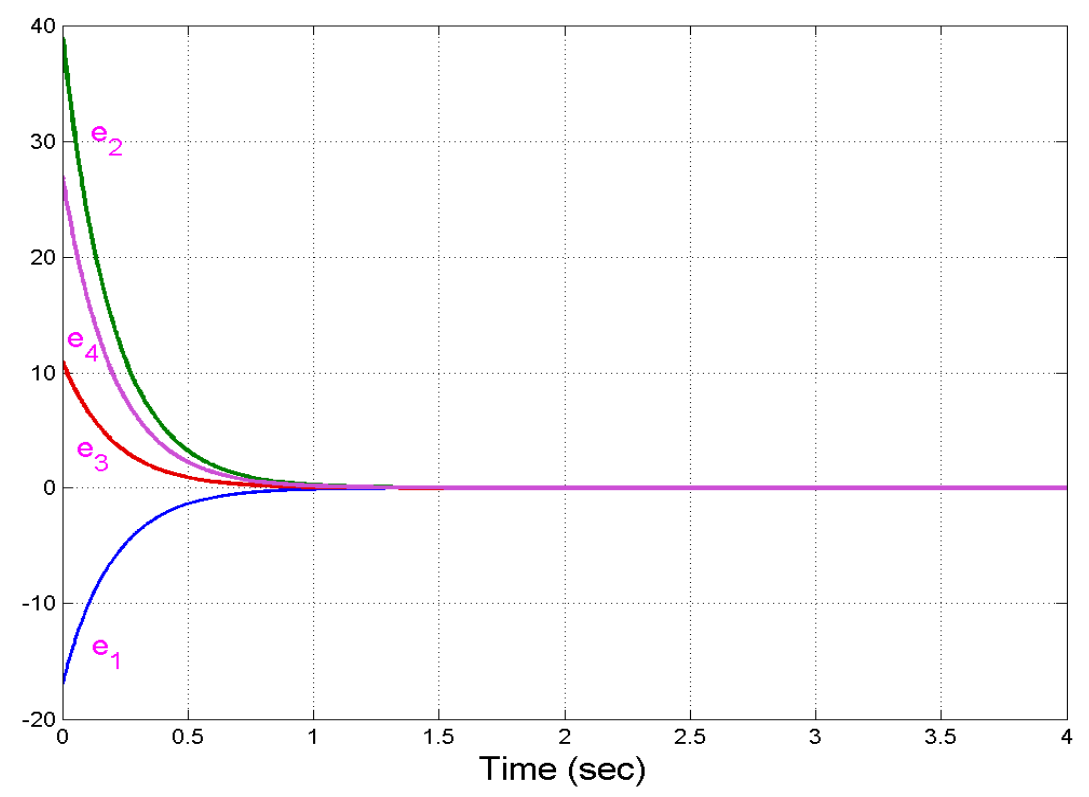

Figure 8. Time-History of the Anti-Synchronization Error 
International Journal of Instrumentation and Control Systems (IJICS) Vol.2, No.2, April 2012

\section{Conclusions}

In this paper, we have used active nonlinear control method and Lyapunov stability theory to achieve anti-synchronization for the identical hyperchaotic Qi systems (2008), identical hyperchaotic Jha systems (2007) and non-identical hyperchaotic Qi and hyperchaotic Jha systems. Since the Lyapunov exponents are not required for these calculations, the active nonlinear control method is very effective and convenient to achieve anti-synchronization for the three master-slave pairs of hyperchaotic systems studied in this paper. Numerical simulations have been shown to illustrate the effectiveness of the anti-synchronization schemes derived in this paper for the hyperchaotic Qi and hyperchaotic Jha systems.

\section{REFERENCES}

[1] Alligood, K.T., Sauer, T. \& Yorke, J.A. (1997) Chaos: An Introduction to Dynamical Systems, Springer, New York.

[2] Rössler, O.E. (1979) “An equation for hyperchaos,” Physics Letters A, Vol. 71, pp 155-157.

[3] Buscarino, A., Fortuna, L. \& Frasca, M. (2009) "Experimental robust synchronization of hyperchaotic circuits," Physica D: Nonlinear Phenomena, Vol. 238, No. 18, pp 1917-1922.

[4] Pecora, L.M. \& Carroll, T.L. (1990) "Synchronization in chaotic systems”, Phys. Rev. Lett., Vol. 64, pp 821-824.

[5] Lakshmanan, M. \& Murali, K. (1996) Nonlinear Oscillators: Controlling and Synchronization, World Scientific, Singapore.

[6] Han, S.K., Kerrer, C. \& Kuramoto, Y. (1995) "Dephasing and burstling in coupled neural oscillators", Phys. Rev. Lett., Vol. 75, pp 3190-3193.

[7] Blasius, B., Huppert, A. \& Stone, L. (1999) "Complex dynamics and phase synchronization in spatially extended ecological system”, Nature, Vol. 399, pp 354-359.

[8] Feki, M. (2003) "An adaptive chaos synchronization scheme applied to secure communication", Chaos, Solitons and Fractals, Vol. 18, pp 141-148.

[9] Murali, K. \& Lakshmanan, M. (1998) "Secure communication using a compound signal from generalized synchronizable chaotic systems”, Phys. Rev. Lett. A, Vol. 241, pp 303-310.

[10] Yang, T. (2004) “A survey of chaotic secure communication systems," International Journal of Computational Cognition, Vol. 2, No. 2, pp 81-130.

[11] Ott, E., Grebogi, C. \& Yorke, J.A. (1990) “Controlling chaos”, Phys. Rev. Lett., Vol. 64, pp 11961199.

[12] Ho, M.C. \& Hung, Y.C. (2002) "Synchronization of two different chaotic systems by using generalized active control", Physics Letters A, Vol. 301, pp 424-428.

[13] Chen, H.K. (2005) "Global chaos synchronization of new chaotic systems via nonlinear control", Chaos, Solitons \& Fractals, Vol. 23, pp 1245-1251.

[14] Sundarapandian, V. (2011) "Global chaos synchronization of four-scroll and four-wing chaotic attractors by active nonlinear control," International Journal on Computer Science and Engineering, Vol. 3, No. 5, pp. 2145-2155.

[15] Sundarapandian, V. (2011) "Global chaos synchronization of Li and Liu-Chen-Liu chaotic systems by active nonlinear control," International Journal of Advances in Science and Technology, Vol. 3, No. 1, pp. 1-12.

[16] Liao, T.L. \& Tsai, S.H. (2000) "Adaptive synchronization of chaotic systems and its applications to secure communications", Chaos, Solitons and Fractals, Vol. 11, pp 1387-1396. 
International Journal of Instrumentation and Control Systems (IJICS) Vol.2, No.2, April 2012

[17] Sundarapandian, V. (2011) "Adaptive control and synchronization of hyperchaotic Cai system", International Journal of Control Theory and Computer Modelling, Vol. 1, No. 1, pp 1-13.

[18] Sundarapandian, V. (2011) "Adaptive synchronization of hyperchaotic Lorenz and hyperchaotic Liu systems”, International Journal of Instrumentation and Control Systems, Vol. 1, No. 1, pp 1-18.

[19] Sundarapandian, V. (2011) "Adaptive control and synchronization of Liu's four-wing chaotic system with cubic nonlinearity," International Journal of Computer Science, Engineering and Applications, Vol. 1, No. 4, pp 127-138.

[20] Sundarapandian, V. \& Karthikeyan, R. (2011) "Global chaos synchronization of Pan and Lü chaotic systems via adaptive control," International Journal of Information Technology, Convergence and Services, Vol. 1, No. 5, pp. 49-66.

[21] Yu, Y.G. \& Zhang, S.C. (2006) "Adaptive backstepping synchronization of uncertain chaotic systems”, Chaos, Solitons and Fractals, Vol. 27, pp 1369-1375.

[22] Wu, X. \& Lü, J. (2003), "Parameter identification and backstepping control of uncertain Lü system," Chaos, Solitons and Fractals, Vol. 18, pp 721-729.

[23] Yang, T. \& Chua, L.O. (1999) "Control of chaos using sampled-data feedback control", Internat. J. Bifurcat. Chaos, Vol. 9, pp 215-219.

[24] Park, J.H. \& Kwon, O.M. (2003) "A novel criterion for delayed feedback control of time-delay chaotic systems”, Chaos, Solitons and Fractals, Vol. 17, pp 709-716.

[25] Sundarapandian, V. (2011) "Global chaos synchronization of four-wing chaotic systems by sliding mode control", International Journal of Control Theory and Computer Modelling, Vol. 1, No. 1, pp 15-31.

[26] Sundarapandian, V. (2011) "Global chaos synchronization of Pehlivan systems by sliding mode control”, International Journal on Computer Science and Engineering, Vol. 3, No. 5, pp 2163-2169.

[27] Sundarapandian, V. (2011) "Sliding mode controller design for the synchronization of ShimizuMorioka chaotic systems", International Journal of Information Sciences and Techniques, Vol. 1, No. 1 , pp 20-29.

[28] Qi, G., Wyk, M.A., Wyk, B.J. \& Chen, G. (2008) “On a new hyperchaotic system”, Physics Lettters A, Vol. 372, pp 124-136.

[29] Jia, Q. (2007) "Hyperchaos generated from the Lorenz system and its control", Physics Letters A, Vol. 366, pp 217-222.

[30] Hahn, W. (1967) The Stability of Motion, Springer, New York.

\section{Author}

Dr. V. Sundarapandian obtained his Doctor of Science degree in Electrical and Systems Engineering from Washington University, St. Louis, USA in May 1996. He is a Professor at the R \& D Centre at Vel Tech Dr. RR \& Dr. SR Technical University, Chennai, Tamil Nadu, India. He has published over 240 refereed international publications. He has published over 160 papers in National and International Conferences. He is the Editor-in-Chief of the AIRCC Journals - International Journal of Instrumentation and Control Systems, International Journal of Control Systems and Computer Modelling, and International Journal of Information Technology, Control

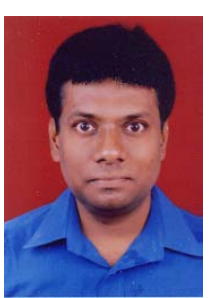
and Automation. His research interests are Linear and Nonlinear Control Systems, Chaos Theory and Control, Soft Computing, Optimal Control, Operations Research, Mathematical Modelling and Scientific Computing. 